\title{
Gutta percha and updated obturating techniques
}

\begin{abstract}
Gutta-percha was first introduced as a restorative material and later developed into an indispensable endodontic filling material. It has become one of the most important materials in endodontics, in its development as a field. Over the years, numerous methods have been advocated to obturate the root canal with gutta-percha and sealer. Hence various forms of gutta-percha are available for usage and the current forms are solid core gutta-percha points, standardized, non- standardized, thermo mechanical compactible gutta-percha, thermo plasticized gutta- percha, solid core system, injectable form, and medicated guttapercha. This article describes its origin, its chemistry, its different phases and properties of gutta-percha in term of using in dentistry. The aim of this study was to elucidate the rheological properties of gutta- percha, and to discuss whether the method using guttapercha is proper for obturating the root canal.
\end{abstract}

Keywords: gutta-percha, chemistry of gutta-percha, different phases of gutta-percha, properties of guttapercha
Volume 8 Issue 2 - 2017

\author{
Karkazi Frantzeska,' Dimitris \\ Christopoulos, ${ }^{2}$ Petros Chondrokoukis ${ }^{2}$ \\ 'Department of Restorative Dentistry, National and \\ Kapodistrian University of Athens, Greece \\ ${ }^{2}$ Department of Restorative Dentistry, Comenius University of \\ Bratislava, Slovakia
}

\section{Correspondence: Karkazi Frantzeska, Department of Restorative Dentistry, National and Kapodistrian University of Athens,Vas. Sofias 105-107, 14561,Athens, Greece,} Email fkarkazi@aol.com

Received: August 17, 2017| Published: September 01, 2017

\section{Introduction}

Gutta-percha is natural latex and obtained from Palaquium gutta and several other trees of East Asia. The latex collected by felling or girdling the tree, is allowed to coagulate and is then washed, purified, and molded into bricks for shipping. Like caoutchouc, gutta-percha is a polyterpene, i.e., a polymer of isoprene (rubber), but, unlike caoutchouc, it is not very elastic; the reason for the difference is that the polymer molecules in gutta-percha have a Tran's structure, whereas those of caoutchouc have a Cis structure (there are isomers). Gutta-percha is an excellent nonconductor and is often employed in insulating marine and underground cables. It is also used for golf-ball coverings, surgical appliances, and adhesives. ${ }^{1}$ The phase transformation is important in thermoplastic obturation techniques. Gutta-percha is soluble in chloroform, eucalyptol, and halothane and less well in turpentine. This property of gutta-percha allows it to be removed for post preparation and in the retreatment of nonhealing cases. Any method manipulating gutta-percha using heat or solvent will result in some shrinkage (1-2\%) of the material. Shrinkage of the core material is not desirable when attempting to seal a canal. Dental gutta- percha is not pure or even mostly gutta-percha. Its major component is zinc oxide (50- 79\%), heavy metal salts (1$17 \%$ ), wax or resin (1-4\%) and only $19-22 \%$ actual gutta- percha. The variations in content are because of different manufacturers and distributors desiring different handling properties. Some formulations are softer than others. Some clinicians choose the brand of guttapercha depending on the technique being used. Compaction with spreaders, condensers or carriers is usually the means used to attempt to compensate for this shrinkage of the core material. In any case, some means of compensation for this shrinkage must be incorporated into the technique being used. An important characteristic of guttapercha and of clinical importance is the fact that when it is exposed to air and light over time it becomes more brittle. Storage of gutta-percha in a refrigerator extends the shelf life of the material. ${ }^{2}$ Chemically pure Gutta-percha exists in two distinctly different crystalline forms ( $\alpha$ and $\beta$ ), that can be interconvertible. Natural gutta-percha is from tree and is $\alpha$ form. However the most commercial available product is in $\beta$ form. During the process of manufacture, if the cooling is done rapidly, ' $\beta$ ' form results. If it is cooled slowly, less than $0.5^{\circ} \mathrm{C}$ per hour, a form is produce. Another unstable form $(\gamma)$ exists, which is amorphous in nature. ${ }^{3}$ Characteristics about different forms:

$\alpha$ : runny, tacky and sticky (lower viscosity)

$\beta$ : solid, compactible and elongatible (higher viscosity)

$\gamma$ : similar to a (unstable) (Figure $1 \&$ Table 1).

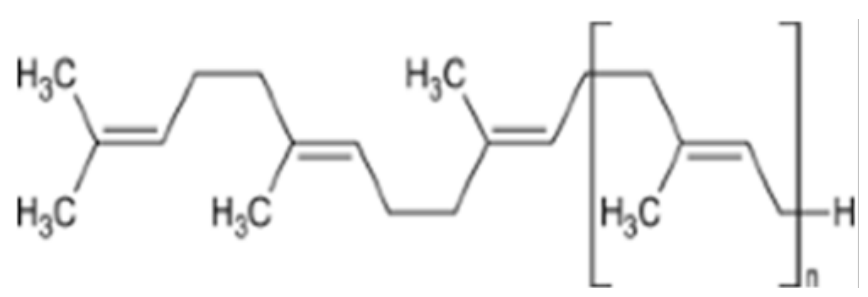

Figure I Chemical form of gutta percha.

Table I Differences between $\alpha$ and $\beta$ form of gutta percha

\begin{tabular}{ll}
\hline Alpha form & Beta form \\
\hline Brittle at room temperature & $\begin{array}{l}\text { Stable and flexible at room } \\
\text { temperature }\end{array}$ \\
$\begin{array}{l}\text { Becomes gluey, adhesive and } \\
\text { highly flowable when heated (low } \\
\text { viscosity) }\end{array}$ & $\begin{array}{l}\text { Becomes less adhesive and } \\
\text { flowable when heated (high } \\
\text { viscosity) }\end{array}$ \\
$\begin{array}{l}\text { Thermoplasticized gutta percha } \\
\text { for warm condensation obturation } \\
\text { technique are in alpha form }\end{array}$ & \\
\end{tabular}

Gutta percha points used for cold condensation obturation techniques are in beta form. Gutta percha exhibits two crystalline transformations when heated from room temperature to 1000 Centigrade. ${ }^{2}$ These transformations can be summarized as follows: 
a. Beta to alpha: occurs between $42^{\circ} \mathrm{C}-49^{\circ} \mathrm{C}$ (this phase transformation is reversible)

b. Alpha to amorphous: occurs between $53^{\circ} \mathrm{C}-59^{\circ} \mathrm{C}$ ADA specification for obturating gutta-percha points.

From then onwards, there was a great surge in the development of root canal therapy as a speciality. Although various cleaning and shaping methods have since been introduced, gutta-percha remains to be the main core material used for root canal fillings. ${ }^{4}$ The studious reader will have noted the use of the word 'compaction' rather than 'condensation'. In 1998, the American Endodontic Association recognized that this was a more appropriate term for the techniques used in obturation. Several techniques have been developed for placing gutta-percha into the root canal system. Nevertheless, the cold lateral compaction of gutta-percha is still the most widely taught, and the technique against which most others are compared. However, as there is a demand for saving teeth with complex pathology and root canal morphology it is sometimes easier to combine the merits of various techniques in a hybrid form to simplify the filling procedure. Studies have shown that these are satisfactory, although not always as easy as lateral compaction to carry out. ${ }^{5}$ Before a root-filling is inserted, it is essential that the canals are dry. Any serous exudate from the periapical tissues indicates the presence of inflammation. Calcium hydroxide may be used as a root canal dressing until the next visit.

\section{Lateral compaction of gutta-percha}

With this technique, the objective is to fill the canal with guttapercha points (cones) by compacting them laterally against the sides of the canal walls. It is a successful technique due to its simplicity, not requiring specific and expensive instruments and low cost. Disadvantages of this technique include risk of void formation, inadequate adaptation of root filling material to the root canal walls and partial filling in certain hard-to-reach areas of the root canal system ${ }^{7}$ The technique requires selection of a master point, usually one size larger than the master apical file, which should seat about $0.5 \mathrm{~mm}$ short of the working length. If the point is loose at working length, then either $1 \mathrm{~mm}$ should be cut from the tip and the point refitted to the canal, or a larger size point selected. ${ }^{8}$ It should be noted that gutta- percha points cannot be as accurately machined as metallic instruments. There may be variance in the size stated, and if a matched point does not fit a prepared canal it may be worth either trying another point from the packet, or fitting the point in a measuring/sizing gauge. ${ }^{9}$ In cold lateral compaction, the master point should exhibit 'tug-back' slightly short of the working lengths. ${ }^{10}$ Once the master point is fitted to length and demonstrates a slight resistance to withdrawal accessory points are then inserted alongside the master point and compacted laterally with a spreader until the canal is sealed. The simplest system of accessory points designates these from $\mathrm{A}$, the finest, through $\mathrm{B}$ and $\mathrm{C}$ to $\mathrm{D}$, the largest. As each point is used the prepared, flared, canal is becoming progressively wider, and the accessory points may therefore be used sequentially from small to large. The resultant filling appears above the access cavity as a spiral, with each point extruded slightly further out of the canal. ${ }^{11}$ There are two main types of spreading instruments for compacting gutta- percha: long-handled spreaders and finger spreaders. The main advantage of a finger spreader is that it is not possible to exert the high lateral pressure that might occur with long-handled spreaders. The chance of a root fracture is reduced and it is therefore a suitable instrument for beginners. ${ }^{12}$ However, Guttapercha points should not be presented to the operator by the surgery assistant with the tips dipped in sealer (Figure 2). ${ }^{13}$

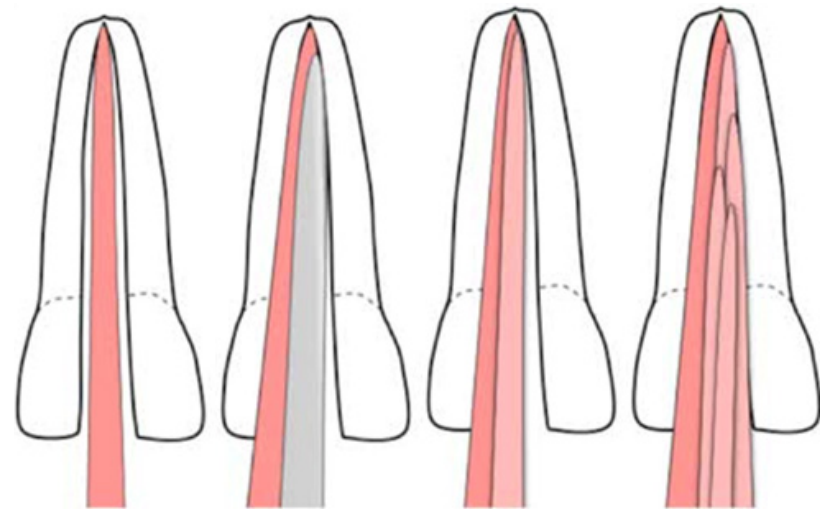

Figure 2 Cold lateral compaction following the placement of master point. ${ }^{27}$

\section{Lateral compaction of warm gutta-percha}

A simple modification to the cold lateral compaction technique is to apply heat to the gutta-percha. The softened material is easier to compact and will result in a denser root filling. However, finger spreaders will not retain heat sufficiently for this procedure, and specially designed heat carriers should be used. The instruments have a sharp tip for lateral compaction, and a blunt plugger tip for limited vertical compaction of the softened gutta-percha. Electrically heated spreaders are also available. It is important that the instruments are only gently warmed. If the spreader is too hot it will melt the guttapercha, which will adhere to the instrument and be withdrawn from the canal. ${ }^{14}$ The main advantages of thermoplasticized gutta percha techniques include better adaptation to root canal complexities, lower risk of void formation and creating a dense filling. ${ }^{15,16}$

\section{Single gutta-percha point and sealer}

With the tendency to preparation techniques of greater taper, gutta-percha points of matching taper may be used. These fit the prepared canal so well that some operators are using a single guttapercha point and sealer. The only advantage of this technique is its simplicity. The disadvantage is that the majority of sealers are soluble. As the canal will not be fully filled in three dimensions, tissue fluids may leach out the sealer over time. This technique cannot therefore be recommended. ${ }^{17}$ However, in difficult anatomical cases it may be necessary to create a custom- fitted cone. A slightly large cone is selected and the apical part softened, either by solvents such as chloroform, rectified turpentine or oil of eucalyptus, or by immersion in hot water. The softened cone is fitted to working length with gentle pressure. The cone is carefully marked for orientation, and the process repeated until a satisfactory fit is obtained. The cone should then be cleaned of all solvents, and the canal obturated using sealer in the usual way. As with all single-cone techniques, if the excess sealer resorbs in the apical tissue fluids, microleakage may allow the ingress of tissue fluids, and failure of the stated criteria of obturation. Really, an attempt should always be made to improve the fit of a single cone with warm or cold lateral compaction of accessory points. ${ }^{18}$

\section{Thematic compaction of gutta-percha}

In 1979, McSpadden devised a handpiece-driven compactor, which is effectively an inverted Hedstroem file. Although no longer made, other similar devices, such as the gutta condenser, are available. The frictional heat from the compactor plasticises the gutta-percha and the blades drive the softened material into the root canal under 
pressure. The main problem found was lack of control over the apical portion of the gutta-percha, which may be extruded through the apex in its softened state. To overcome this problem, the technique was modified by Tagger, who recommended laterally condensing a master point and two or three accessory points, and then using the condensor to plasticise the gutta-percha in the coronal part of the canal. The laterally compacted material in the apical half effectively prevents any apical extrusion. ${ }^{19}$ The technique is particularly useful for the rapid and effective obturation of the coronal part of a root canal after placement of an accurate apical seal.

\section{Heated gutta-percha carriers}

Several manufacturers now supply these devices. Alpha-phase gutta-percha is attached to a rigid carrier, in a variation of the technique originally described by Johnson in 1978. Most carriers are now plastic. The excess material is removed, and the carrier remains in the canal as a central core. The softened gutta-percha flows well in to canal aberrations, fins, etc., giving very good three-dimensional obturation. Success depends, as with all techniques, upon thorough canal cleaning and shaping. The carriers have a $4 \%$ taper, and an underprepared canal will be difficult if not impossible to obturate to working length with these devices. A range of sizes is presented, and most systems employ a method of ensuring the fit of the device before obturation is commenced. This may either be a blank carrier with no gutta-percha attached, or preferably a file of the same dimensions as the carrier. The apical preparation may then be refined to ensure an accurate fit of the device. ${ }^{20}$ The canal should be cleaned and dried, and a very fine coating of sealer applied to the canal orifice only. Excess sealer may be extruded under hydraulic pressure through the apical foramen, with resultant pain and inflammation. In the meantime the rubber stop on the selected device is set to working length, and the device placed in a special oven to soften the gutta-percha. When ready, the device should be swiftly and smoothly inserted to working length, and held in place for a few seconds. Using a high-speed bur the excess carrier may be sectioned and removed from the canal orifice, and a plugger used to compact the gutta-percha in this area. A layer of resinmodified glass ionomer completes the obturation. Some carriers are manufactured with a U-shaped cross-section to facilitate removal with a drill should retreatment be necessary. However, although it may be possible to drill out the carrier, this technique may not be appropriate if a post and core may be indicated in the future (Figure 3). ${ }^{21}$

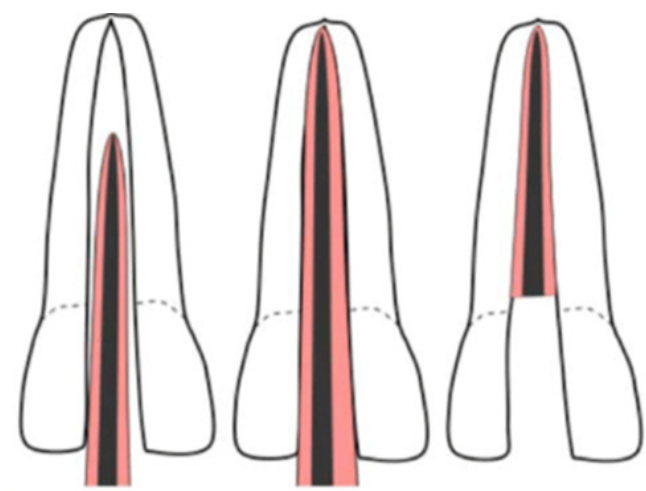

Figure 3 Heated gutta-percha carriers. ${ }^{27}$

\section{Vertical compaction of warm gutta-percha}

Heated gutta-percha has been shown to flow extremely well into all canal irregularities. It is particularly useful in situations such as internal resorption, $\mathrm{C}$ - shaped canals, and those with fins or webs. As referred to earlier, when the smear layer is removed the gutta-percha has been shown to penetrate dentine tubules. This technique is now considered the gold standard for endodontic obturation. The principle of vertical compaction of increments of warm gutta-percha was first described by Schilder in 1967. Although delivering excellent results, the method was difficult to master and time-consuming. ${ }^{22}$ The state of the art at present is the method first described by Buchanan employing the System-B heat source which delivers a precise heat to the tip of the plugger. A non-standardised $(4 \%, 6 \%$ or feathered tip) guttapercha cone is carefully fitted to the canal. Using a selected plugger, a continuous wave of heat is applied to soften and downpack a cone, resulting in very well-compacted obturation of the apical portion of the canal. The remainder of the canal may be obturated by further increments, or by another method. ${ }^{23}$ When the ring on the handpiece is pressed as shown the tip of the plugger is immediately heated to the temperature selected (Figure 4).

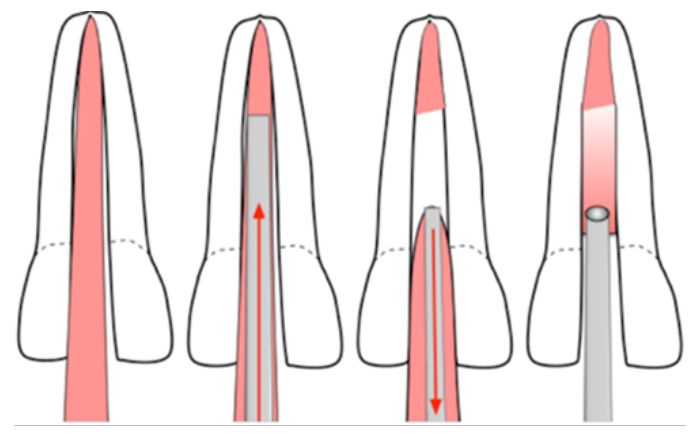

Figure 4 Vertical compaction of warm gutta-percha. ${ }^{27}$

\section{Injectable gutta-percha}

Devices for injecting softened gutta-percha have been available for some time, but in the past have suffered from techniques which led to difficulty in accurate apical placement. The latest of these, the Obtura-II, has recently gained acceptance by endodontists. The machine resembles a glue-gun. Pellets of alpha-phase gutta-percha are softened at about $200^{\circ} \mathrm{C}$ in the handpiece, and extruded through a heated silver needle. A wide, well-prepared canal is a prerequisite. Although the manufacturers describe a procedure for total obturation of a root canal, apical control can be difficult. The machine has become accepted for two specific procedures. ${ }^{24}$

\section{Coronal back-filling}

The previously described System-B achieves an excellent and controlled obturation of the apical 5-7 mm of the root canal. At this point the canal is quite wide, and can accept the tip of the Obtura's needle. A film of sealer is applied to the canal wall. The machine is heated to $200^{\circ} \mathrm{C}$. A small amount of the warm gutta-percha should be extruded to warm the needle and discarded. The needle is then quickly introduced to the canal. If this part of the protocol is not followed, a void may result between the two parts of the filling. The trigger is activated and thermoplasticised gutta-percha extruded into the canal, gently pushing the needle out. Once the canal is filled conventional pluggers may be used to compact the gutta-percha, which is finally sealed with glass ionomer as usual. ${ }^{25}$

\section{Discussion}

Gutta-percha used for endodontic obturation purposes are both, alpha and beta forms, $\mathrm{b}$ form of gutta-percha was used for improved 
stability, hardness and reduced stickiness. But the phase gutta-percha with low viscosity will flow with less pressure or stress, create a more homogenous filling. There are different forms of gutta-percha are available: Solid core gutta-percha points [standardized and non- standardized points (beta phase)], are used with cold lateral condensation with warm vertical compaction. Thermoplasticized gutta-percha usually available in injectable form (alpha phase). Thermoplaticized gutta-percha systems provide special heaters which heats the gutta-percha till the temperature when it flows. Several filling techniques have been developed for placing gutta-percha into the root canal system. The cold lateral compaction of gutta-percha is still the most widely taught, and the technique against which most others are compared.

a. Lateral compaction of gutta-percha: with this technique, the objective is to fill the canal with gutta-percha points (cones) by compacting them laterally against the sides of the canal walls.

b. Lateral compaction of warm gutta-percha: a simple modification to the cold lateral compaction technique is to apply heat to the gutta- percha.

c. Single gutta-percha point and sealer: with the tendency to preparation techniques of greater taper, gutta-percha points of matching taper may be used. These fit the prepared canal so well that some operators are using a single gutta-percha point and sealer. The only advantage of this technique is its simplicity. The disadvantage is that the majority of sealers are soluble. This technique cannot therefore be recommended.

d. Thermatic compaction of gutta-percha: the frictional heat from the compactor plasticises the gutta-percha and the blades drive the softened material into the root canal under pressure.

e. Heated gutta-percha carriers: alpha-phase gutta-percha is attached to a rigid carrier. Most carriers are now plastic. The excess material is removed, and the carrier remains in the canal as a central core. The softened gutta-percha flows well in to canal aberrations.

f. Vertical compaction of warm gutta-percha: It is particularly useful in situations such as internal resorption, C-shaped canals, and those with fins or webs. This technique is now considered the gold standard for endodontic obturation. Although delivering excellent results, the method was difficult to master and time-consuming.

\section{Conclusion}

The lateral condensation technique, which fills using one main point and a number of accessory points placed in the root canal space with sealer, has been widely accepted and used clinically for a long time. Additionally, a number of techniques for root canal filling using gutta-percha have been developed, and most of them make use of its properties of thermoplasticity and solubility with organic solvent From then on, there was a great surge in the development of root canal therapy as a speciality. Although various cleaning and shaping methods have since been introduced, gutta-percha remains to be the main core material used for root canal fillings.

\section{Funding}

None.

\section{Acknowledgments}

None.

\section{Conflicts of interest}

Author declares that there is no conflict of interest.

\section{References}

1. Bowman C, Baumgartner JC. Gutta-percha obturation of lateral grooves and depressions. J Endod. 2002;28(3):220-223.

2. Prakash R, Gopikrishna, Kandaswamy. Gutta percha-An Untold story. Endodontology. 2005;17(2):32-36.

3. Schilder H, Goodman A, Aldrich W. The Thermo Mechanical properties of Gutta Percha. III. Determination of phase transition temperatures for Gutta Percha. Oral Surg Oral Med Oral Pathol. 1974;38(1):109-114.

4. Combe EC, Cohen BD, Cummings K. Alpha and beta-forms of Guttapercha in products for root canal filling. Int Endod J. 2001;34(6):447-451.

5. Goodman A, Schilder H, Aldrich W. The thermomechanical properties of gutta- percha II: the history and molecular chemistry of gutta-percha. Oral Surg Oral Med Oral Pathol. 1974;37(6):954-961.

6. Evans JT, Simon JHS. Evaluation of the apical seal produced by injected thermoplasticized gutta-percha in the absence of smear layer and root canal sealer. J Endod. 1986;12(3):101-107.

7. Peters CI, Sonntag D, Peters OA. Homogeneity of root canal fillings performed by undergraduate students with warm vertical and cold lateral techniques. Oral Surg Oral Med Oral Pathol Oral Radiol Endod. 2010;110(3):e41-e49.

8. Clinton K, Van Himel T. Comparison of a warm gutta-percha obturation technique and lateral condensation. J Endod. 2001;27(11):692-695.

9. Inan U, Aydemir H, Taşdemir T. Leakage evaluation of three different root canal obturation techniques using electrochemical evaluation and dye penetration evaluation methods. Aust Endod J. 2007;33(1):18-22.

10. Gutmann JL. Adaptation of injected thermoplasticised gutta percha in the absence of the dentinal smear layer. Int Endod J. 1993;26(2):87-92.

11. Carrotte P. Endodontics: Part 8 Filling the root canal system. BDJ. 2004;197:667-672.

12. Schilder H, Goodman A, Aldrich W. The thermomechanical properties of gutta- percha. Part V. Volume changes in bulk gutta-percha as a function of temperature and its relationship to molecular phase transformation. Oral Surg Oral Med Oral Pathol. 1985;59(3):285-296.

13. Cohen BD, Combe EC, Lilley JD. Effect of thermal placement techniques on some physical properties of gutta-percha. Int Endod J. 1992;25(6):292-296.

14. Carrotte P. Endodontics: Part 8 Filling the root canal system. British Dental Journal. 2004;197:667-672.

15. Lea CS, Apicella MJ, Mines $\mathrm{P}$, et al. Comparison of the obturation density of cold lateral compaction versus warm vertical compaction using the continuous wave of condensation technique. J Endod. 2005;31(1):37-39.

16. Collins J, Walker MP, Kulild J, et al. A comparison of three guttapercha obturation techniques to replicate canal irregularities. $J$ Endod. 2006;32(8):762-765.

17. Emmanuel S, Shantaram K, Sushil KC, et al. An In Vitro Evaluation and Comparison of Apical Sealing Ability of Three Different Obturation Technique-Lateral Condensation, Obtura II, and Thermafil. J Int Oral Health. 2013;5(2):35-43. 
18. Wu MK, Bud MG, Wesselink PR. The quality of single cone and laterally compacted gutta-percha fillings in small and curved root canals as evidenced by bidirectional radiographs and fluid transport measurements. Oral Surg Oral Med Oral Pathol Oral Radiol Endod. 2009;108(6):946-951.

19. Marciano J, Michailesco P, Charpentier E, et al. Thermomechanical analysis of dental gutta-percha. J Endod. 1992;18(6):263-270.

20. Tagger M, Gold A. Flow of various brands of gutta-percha cones under in vitro thermomechanical compaction. J Endod. 1988;14(3):115-120.

21. Wu MK, Kastáková A, Wesselink PR. Quality of cold and warm guttapercha fillings in mandibular premolars. Int Endod J. 2001;34(6):485-491.

22. Aminsobhani M, Ghorbanzadeh A, Sharifian MR, et al. Comparison of Obturation Quality in Modified Continuous Wave Compaction, Continuous Wave Compaction, Lateral Compaction and Warm Vertical Compaction Techniques. J Dent (Tehran). 2015;12(2):99-108.
23. Weller RN, Koch KA. In vitro radicular temperatures produced by injectable thermoplasticized gutta-percha. Int Endod J. 1995;28(2):86-90.

24. Saunders EM. In vivo findings associated with heat generation during thermomechanical compaction of gutta-percha. 1. Temperature levels at the external surface of the root. Int Endod J. 1990;23(5):263-267.

25. Olson AK, MacPherson MG, Gary R Hartwell, et al. An in vitro evaluation of injectable thermoplasticized guttapercha, glass ionomer, and amalgam when used as retrofilling materials. J Endod. 1990;16(8):361-364.

26. Madke L. Importance of coronal seal: Preventing coronal leakage in endodontics. J Res Dent. 2016;4(3):71-75.

27. Darcey J, Roudsari RV, Jawad S, et al. Modern Endodontic Principles. Part 5: Obturation. Dent Update. 2016;43(2):114-126. 